\title{
Passive Profiling and Natural Interaction Metaphors for Personalized Multimedia Museum Experiences
}

\author{
Svebor Karaman, Andrew D. Bagdanov, Gianpaolo D'Amico, Lea Landucci, \\ Andrea Ferracani, Daniele Pezzatini, and Alberto Del Bimbo \\ Media Integration and Communication Center (MICC) \\ University of Florence, Florence, Italy \\ \{svebor.karaman, andrea.ferracani, daniele.pezzatini\}@unifi.it, \\ \{bagdanov, damico, delbimbo\}@dsi.unifi.it, lea.landucci@gmail.com \\ http://www.micc.unifi.it/vim/people
}

\begin{abstract}
Museums must balance the amount of information given on individual pieces or exhibitions in order to provide sufficient information to aid visitor understanding. At the same time they must avoid cluttering the environment and reducing the enjoyment of the exhibit. Moreover, each visitor has different interests and each might prefer more (or less) information on different artworks depending on their individual profile of interest. Finally, visiting a museum should not be a closed experience but a door opened onto a broader context of related artworks, authors, artistic trends, etc. In this paper we describe the MNEMOSYNE system that attempts to provide such a museum experience. Based on passive observation of visitors, the system builds a profile of the artworks of interest for each visitor. These profiles of interest are then used to personalize content delivery on an interactive table. The natural user interface on the interactive table uses the visitor's profile, a museum content ontology and a recommendation system to personalize the user's exploration of available multimedia content. At the end of their visit, the visitor can take home a personalized summary of their visit on a custom mobile application. In this article we describe each component of our approach as well as the first field trials of our prototype system built and deployed at our permanent exhibition space at Le Murat 11 in the city of Florence.
\end{abstract}

Keywords: Computer vision, video surveillance, cultural heritage, multimedia museum, personalization, natural interaction.

\section{Introduction}

Modern museums are awash in physical and digital content that they struggle to catalog, to maintain, to manage, and - most importantly - to deliver in meaningful ways to the museum-going public. Each visitor would like to easily access different aspects of the massive amount of available information. To address this

1 http://www. lemurate.comune.fi.it/lemurate/

A. Petrosino, L. Maddalena, P. Pala (Eds.): ICIAP 2013 Workshops, LNCS 8158, pp. 247-256, 2013.

(C) Springer-Verlag Berlin Heidelberg 2013 


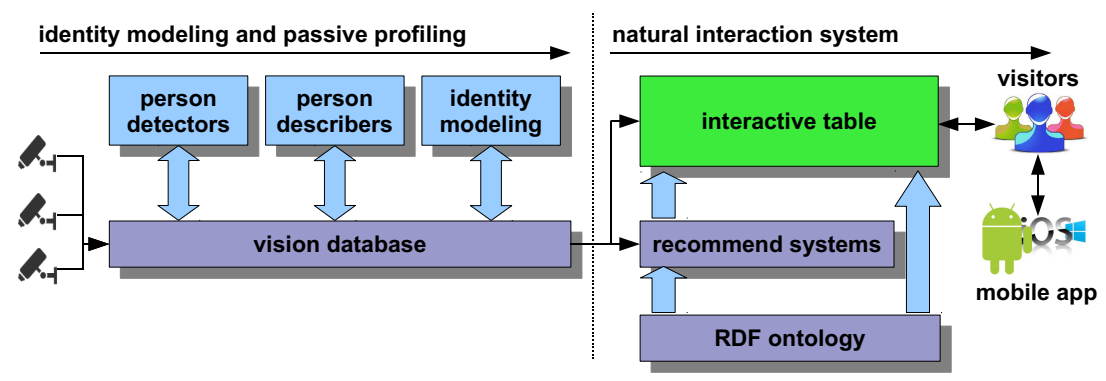

Fig. 1. An overview of the MNEMOSYNE architecture

issue most research has focused on providing personalized access through handheld devices carried by visitors [15] and possibly offering some sort of augmented reality experience 113 . However, the use of mobile devices is intrusive to the museum experience as it changes the way the visitor behaves with respect to the museum. It also requires active participation of the user in front of each artwork of interest.

Museum exhibits are often designed out of the need to target a sort of "common denominator" visitor. This necessity arises from the difficulty in understanding a priori the interests of individual visitors in order to customize content delivery in a meaningful way. User interest modeling for personalization has been addressed in [12] where the user inputs his interests both on the museum website and inside the museum in order to create a "virtuous circle" of online and offline visits. In [8, the authors propose to model user interest based on his displacement in the museum environment in order to personalize audio content delivery via a specific audio guide given to each user. This last approach, like the MNEMOSYNE system we describe in this article, differs from the global trend as it does not require explicit input from the user.

MNEMOSYNE is a three-year research project 2] studying techniques for passively observing museum visitors [9] in order to build profiles of interest for personalizing multimedia content delivery (see figure 11). Aiding the delivery of multimedia content, an ontology that models museum content is used to infer connections between visitor interest and works on display, works located in other collections, and works of broader interest. This knowledge model, along with a statistical recommendation system, is used to drive a natural user interface on a large format interactive table. The user interface allows visitors to explore digital museum content personalized to their own interests, and a mobile application allows them to download content of interest they have explored during their visit to a smartphone or tablet.

In the next section we describe the passive profiling system in detail. In section 3.1 we detail the two types of recommendation systems we have experimented with, and in section 3.2 we describe the natural interaction user interface used to deliver personalized content. Finally, in section 4 we discuss the first field trials of our prototype system. 


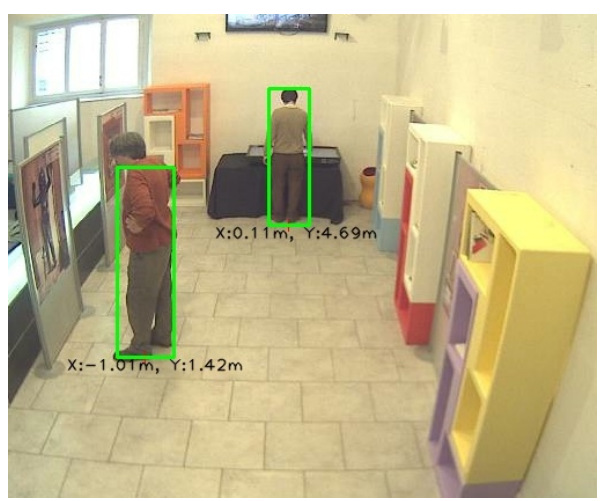

(a)

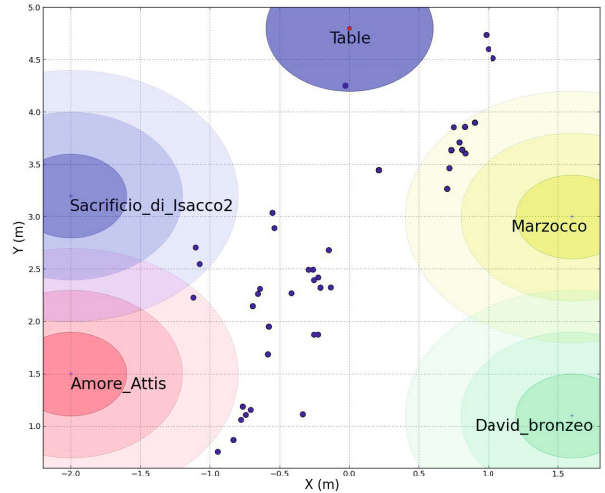

(b)

Fig. 2. (a) Example of frame with detections (b) Detection map with artwork sphere of influence for one visitor model

\section{Passive Interest Profiling}

Here we detail each step of the MNEMOSYNE passive visual profiling approach. First, a pre-processing step is performed to map the artistic content and physical properties of the museum. Then, fixed cameras are used to observe visitors as they visit the museum. Our aim is to maintain a record of what each visitor has observed during their visit and to build a profile of interest him.

\subsection{Mapping the Physical Museum}

Most museums are often already equipped with a set $\mathcal{C}$ of fixed cameras installed for surveillance purposes, and the MNEMOSYNE system is designed to exploit these already-installed cameras. We calibrate each camera $c$ to a common ground plane. A simple tool allows an operator to estimate the homography $\mathcal{H}_{c}$ from each camera image plane to the ground plane with a few mouse clicks [7.

Given the homography $\mathcal{H}_{c}$, an operator can easily input the position on the ground plane of each artwork of interest by simply clicking once in the camera view where the artwork sits on the ground or on the position on the floor below the artwork. A sphere of influence is associated with each artwork, defined as a bi-dimensional Gaussian of mean equal to the ground position of the artwork and variances in $x$ and $y$ dimensions defined by the operator. These variances depend on the structure of the museum as well as the artwork scale.

\subsection{Identity Modeling}

On the video stream corresponding to camera $c \in \mathcal{C}$, we run a pedestrian detector [4] in order to obtain a set of $N$ person bounding boxes. The bounding boxes are then described with a number of visual, temporal and spatial descriptors (the 
person describers module in figure 11). The descriptor of a person bounding box is defined as:

$$
d_{i}=\left\{\mathbf{d}_{i}^{a}, \mathbf{d}_{i}^{s}, d_{i}^{t}, d_{i}^{c}\right\}, \text { for } i \in\{1, \ldots, N\}
$$

where $\mathbf{d}_{i}^{a}$ is an appearance descriptor consisting of RGB and HS color histograms computed on overlapping horizontal stripes and the HoG (Histogram of Oriented Gradients) descriptor [6] as proposed for person re-identification in [10], $\mathbf{d}_{i}^{s}=$ $\left(d_{i}^{x}, d_{i}^{y}\right)$ is the absolute position of the person detection on the ground plane, $d_{i}^{t}$ is an integer timestamp, and $d_{i}^{c}$ is an index indicating that the detecion comes from camera $c$. All video streams are synchronized so that $d_{i}^{t}$ and $d_{j}^{t}$ are comparable.

The fundamental step in passive profiling is associating the detections $D=$ $\left\{d_{i} \mid i=1 \ldots N\right\}$ to one another to form groups representing individual visitors in the museum. Algorithm 1 details the procedure used to build identity models and to associate detections to them. This algorithm relies on the computation of the distance between a model cluster $m_{j}$ and a detection description $d_{i}$ which takes into account the appearance and all spatio-temporal information available. Precisely, the distance between a description $d_{i}$ and model $m_{j}$ is computed as:

$$
\begin{aligned}
\operatorname{dist}\left(m_{j}, d_{i}\right) & =(1-\alpha-\beta) \times\left\|\mathbf{m}_{j}^{a}-\mathbf{d}_{i}^{a}\right\|_{2} \text { (appearance contribution) } \\
& +\alpha \times \operatorname{dist}_{w}\left(\mathbf{m}_{j}^{s}, \mathbf{d}_{i}^{s}, w_{s}\right) \quad \text { (spatial contribution) } \\
& +\beta \times \operatorname{dist}_{w}\left(m_{j}^{t}, d_{i}^{t}, w_{t}\right) \quad \text { (temporal contribution) }
\end{aligned}
$$

where $\operatorname{dist}_{w}(x, y, w)$ is the windowed L2 distance:

$$
\operatorname{dist}_{w}(x, y, w)=\min \left(\frac{\|x-y\|_{2}}{w}, 1\right) .
$$

The parameters $w_{s}$ and $w_{t}$ are, respectively, the spatial and temporal window around observations. The weights $\alpha$ and $\beta$ control the contribution of spatial and temporal distances, respectively, to the overall distance calculation and are defined such that $\alpha, \beta \in[0,1]$ and $\alpha+\beta<1$. A detection is associated with a model if its distance to the model is less than $\delta$. The system must accumulate at least $\tau$ detections in a temporary model before promoting it to a real one. The appearance of a model $\mathbf{m}_{j}^{a}$ is computed as a running average of the detections associated to it, while the position and time information are those of the last matched detection. Whether a model is active is determined by the last associated detection time. Note that we also forbid multiple associations from one camera at the same timestamp to the same model.

\subsection{Interest Profiling}

Each visitor's interest profile is built on-the-fly when the visitor enters the interactive table area (see figure 2b) and is sent to the interactive table. Every detection associated with the visitor's model contributes to each artwork according to its proximity to the artwork sphere of influence. If the visitor leaves the interactive area, goes and sees some other artworks and comes back to the table, his interest profile will be updated. 


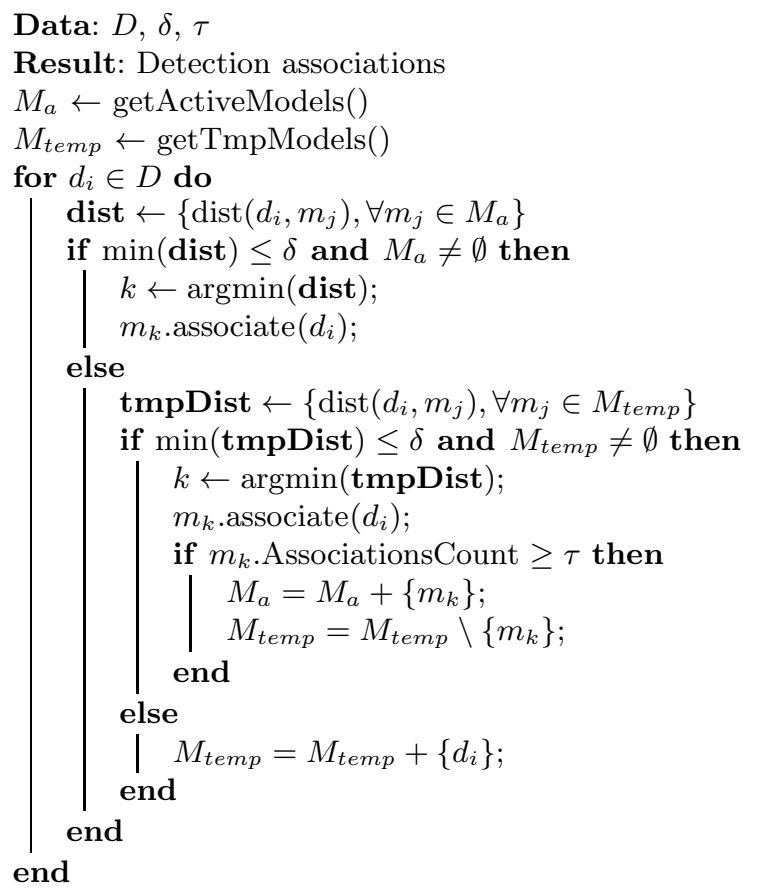

Algorithm 1. Detection association algorithm

\section{The Augmented Museum Experience}

The second stage of the museum visit consists of entering an area equipped with an interactive tabletop display in which users can have a seamless multimedia experience and increase their knowledge of art and cultural heritage. Thanks to the natural interaction with the table, they can explore and select in-depth and related information about each artwork according to the suggestions given by the recommendation systems, further personalizing the profile of interest built passively during their visit. Their favorite artworks and related multimedia content can then be transferred to a mobile device via a dedicated application.

\subsection{Recommendation Systems}

The MNEMOSYNE prototype uses two solutions for generating content recommendations to users: a Knowledge-based and an Experience-based system. These modules have been developed as web servlets which expose several web services accessible via a Representational State Transfer (REST) interface.

Knowledge-Based Recommendation. As a use case we chose one of the most famous museums of Florence: the National Museum of Bargello. In particular, we focus on a subset of 8 monitored artworks from the 70 artworks displayed in the Sala di Donatello. The MNEMOSYNE Semantic Search Engine 
exploits the potential of the Semantic Web through an RDF (Resource Description Framework) ontology that models not only instances of these artworks, but also places, events, historical curiosities and other artworks from Florence and all over the world, and their relations to the 8 chosen artworks. Our ad-hoc ontology implements six different entities: artist, artwork, category, museum/place and story. Every artwork instance is equipped with information about its creator, meanings, materials, techniques and historical context (all represented by instances of their own). Each artwork entity is also accompanied by a variety of multimedia content and has different thematic links to other artworks or stories. SPARQL queries are used to query subgraphs of data from the ontology, providing different views on the data model: subgraphs of artwork data, stories related to artworks, and resources related by tags or stories.

Experience-Based Recommendation. The MNEMOSYNE Recommendation Engine uses two types of recommendation algorithms. Recommendation algorithms try to solve a problem of prediction and give the system the ability to suggest to users items that they are more likely to find interesting. The Recommender Engine implements metrics based on both user similarity and item similarity. The data model consists of preferences stored as triples in a database table containing the following fields: the user ID, the item ID, and a value, assigned by the MNEMOSYNE passive visual profiling module, expressing the strength of the user preference for the item. From this information we compute which users or items are more similar. Both similarity metrics, user based and item based, make use of the same components: a data model, a metric of similarity, a notion of proximity (i.e. a neighborhood of users or items) and an algorithm that predicts values of preference weighting them differently according to the similarity metric. The Recommendation Engine uses Euclidean distances: a greater distance indicates a lower similarity. The system makes use of the Mahout library2, the state of the art for machine learning on big data.

\subsection{The Natural Interaction Tabletop System}

When a visitor approaches the table, he is detected by the camera which triggers the tabletop system and the computer vision system to exchange data about his profile of interest which had been built during their interaction with the physical artworks. Initially the display of the tabletop shows a language selector and then an interactive tutorial as a carousel of images.

The metaphor used for the user interface is based on the idea of the hidden museum waiting to be unveiled, an all-digital environment where visitors can go beyond the artworks level of the physical museum. The tabletop provides an augmented perspective of the museum thanks to the interest profile estimated by the computer vision system, the recommendation systems, and via the adoption of natural interaction paradigms. With the natural interaction interface visitors can discover new, interesting information and resources, and then collect them for future inspection. The main user interface adopts principles derived from

2 http://mahout.apache.org 


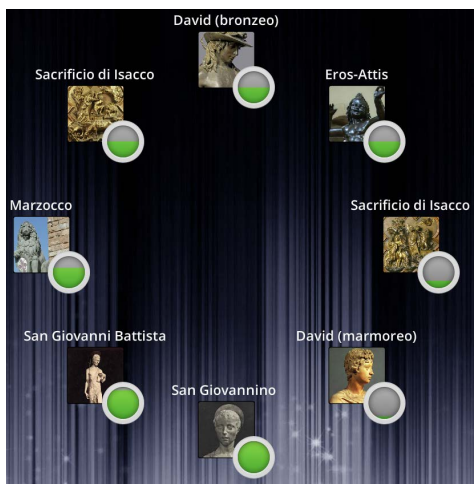

(a)

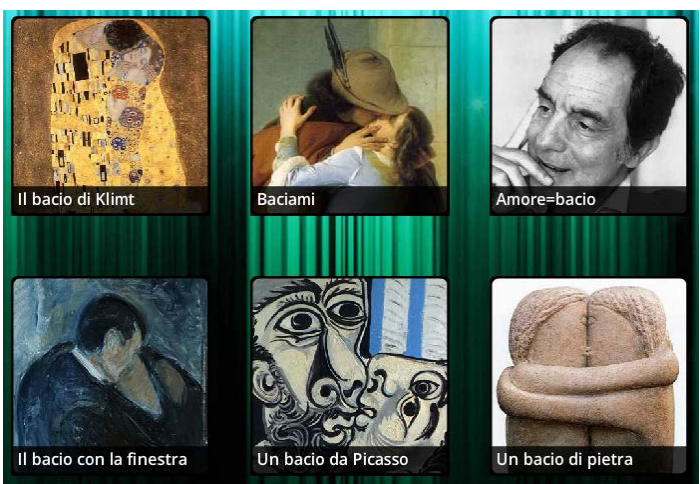

(b)

Fig. 3. (a) Detail of the artwork level: the artworks of the museum are represented with the original title, a thumbnail and a circular symbol visualizing the amount of interest showed by the current user during the visit. (b) Detail of the insights space in the related resources level: information related thematically to the selected artwork.

Information Visualization and consists of two levels of navigation: the artworks and the related resources.

The Artworks Level. visualizes artworks of the museum for which the visitor has shown the highest level of interest (see figure [3a) based on the profile created by the computer vision system. When the user touches an artwork an animation starts in order to trigger the following actions: the space containing the unselected artworks moves up vertically off the screen, the selected artwork moves to the middle left and a new space of information comes up from the bottom.

The Related Resources Level. follows the selection of an artwork and consists of a structured environment in which the visitor can navigate the multimedia content related to this artwork. Related resources are organized in four different spaces (stories, secrets, recommendations, insights) according to their relations to the artwork based on the recommendation system and the ontology structure:

- stories: stories directly related to the artwork in the ontology;

- secrets: resources related to the artwork and its related stories in the ontology according to the knowledge-based recommendation system;

- recommendations: similar artworks according to the experience recommendation system using the visitor profile;

- insights: resources related by tags or stories to the artwork according to the knowledge-based recommendation system.

The user can navigate these spaces via a textual menu at the bottom of the display. In each space details are represented in a different layout: the background color, shape and arrangement of interactive items are all adjusted in order to highlight the difference between the visualized data and to provide the user 


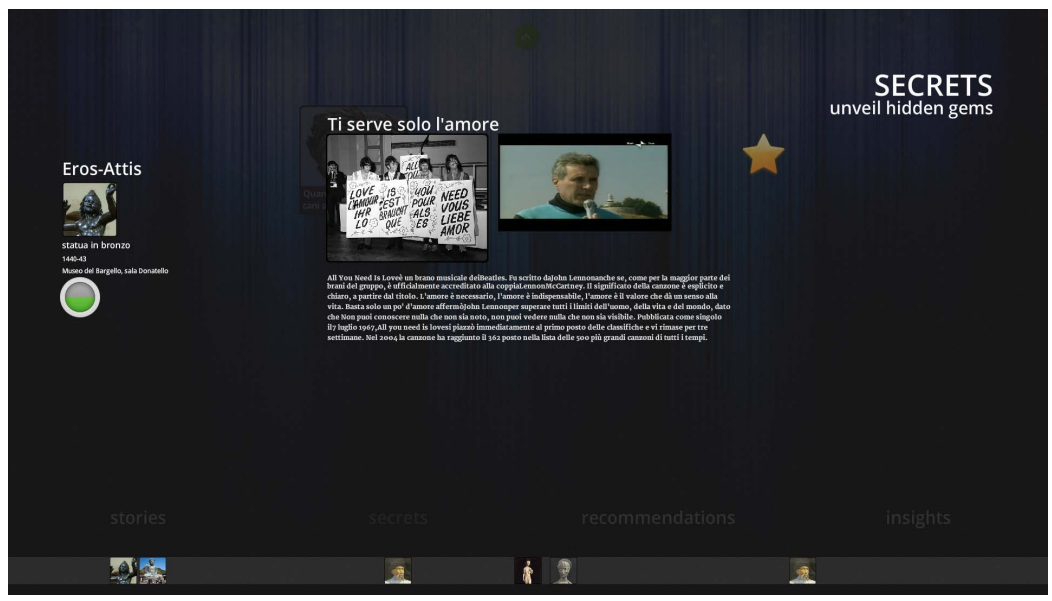

Fig. 4. The information level: when the user selects an item from one space, multimedia content is displayed in the center of the screen. A star element can be touched in order to collect the item in the space at the bottom of the screen. The selected artwork is visualized on the left of the screen with its title, a thumbnail, location and level of visitor interest.

a pleasant experience while navigating in the information space. The insights space is illustrated in figure $3 \mathrm{~b}$. In each space, if the user touches one of the visible items an information window composed of several multimedia contents is triggered, see figure 4. The visitor is able to collect the most interesting resources for later use via the mobile system.

\subsection{Mobile System}

The main goal of the MNEMOSYNE mobile application, shown in figure 5, is to collect personalized digital content displayed in the interactive tabletop interface. Unlike [511], the mobile app is intended to be used at the end of the visit and not as an interactive device throughout the museum tour. It was developed using the Adobe AIR framework and can therefore be installed on devices running iOS (iPhone, iPad) or Android.

Once installed on the visitor's device, the application allows the user to scan a QR code displayed on the interface of the interactive table. This transfers the unique identifier of the user to the mobile device. The application queries the MNEMOSYNE database to retrieve the user's favorite artworks, generated both through the passive profiling module and from his interaction on the tabletop surface. The user then has access to in-depth information about individual and related artworks or resources in the MNEMOSYNE dataset. In particular, the user can visualize a collection of points on a map of interest suggested by the recommendation system taking into account the user's profile and current geolocalization. The latter functionality allows us to extend the personalized user experience of the visit from an indoor to an outdoor scenario. 

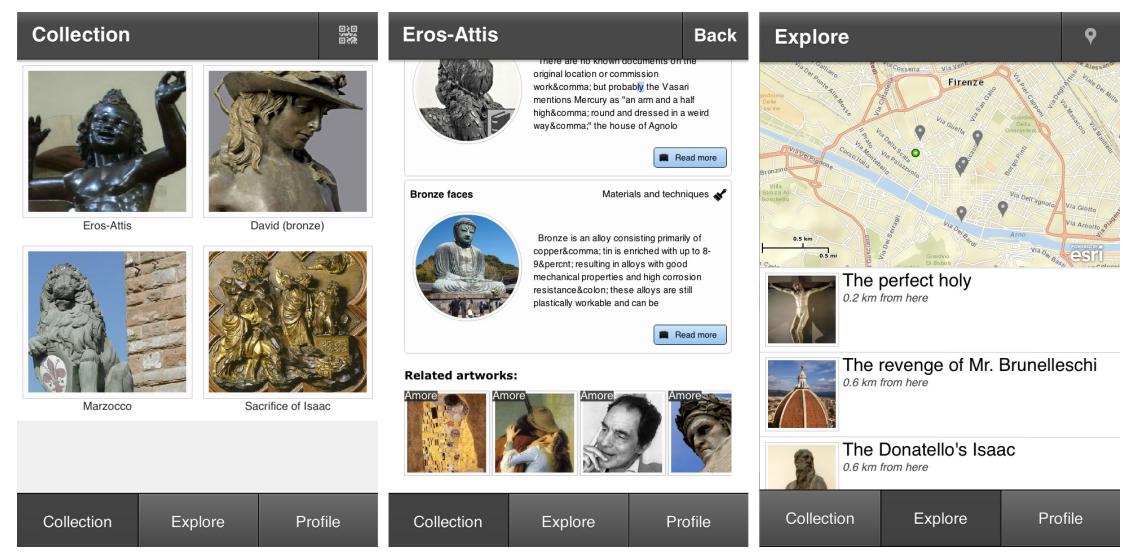

Fig. 5. The mobile application. Left: user's favorite artworks; Center: in-depth information on the artwork; Right: map of suggested points of interest.

\section{$4 \quad$ Field Trials and Ongoing Work}

We have installed a prototype of the MNEMOSYNE system in a permanent exhibition space at Le Murate in the historical center of Florence. For this installation, we printed four high resolution images of artworks from the Donatello Room and used a single surveillance camera to capture images of visitors observing the artworks as well as interacting with the table. Some detections obtained are shown in figure $2 \mathrm{a}$. For this installation the parameters were set manually $\left(\alpha=\beta=0.2, w_{s}=5 \mathrm{~m}, w_{t}=80\right.$ frames, $\delta=0.75$ and $\left.\tau=10\right)$, but given a training set of annotated detections they could be easily learned. Two critical issues were evident when running the system continuously for several hours: ensuring that the system does not lag and that profile messages are thus sent in a timely fashion; and limiting the confusion between visitors since, when observing for several hours, it is very likely that several persons will have similar appearance.

Lag is mostly due to the detection process which is computationally onerous, and we dedicated an 8-core computer to this task and limited the frame rate to 5 frames per second. Moreover, we implemented a lag monitor that considers a maximum allowed lag (set to 5 seconds) and discards frames until the lag falls within the allowed range. To limit confusion between visitors with similar appearance across several hours of observation we limit the association of detections to visitor models that were "active", i.e. ones with which at least one detection was associated in the previous 10 minutes. These proposed solutions are the first necessary steps towards bringing MNEMOSYNE to real museum.

\section{Conclusions}

In this paper we detailed our proposal to enhance and personalize museum visits. Our system makes use of passive observation to estimate each visitor's interest. 
This interest profile is then used conjointly with a recommendation system to provide personalized content delivery through a natural interaction interface. MNEMOSYNE is operational and has been tested, but in order to determine the efficiency of the approach several aspects must be evaluated with further experiments, user studies, and also potentially on how the suggested resources impact visitor visits. We are also interested in using the mobile application as a bridge between different museums adopting MNEMOSYNE, by suggesting artworks of interest in other museums. These are our main objectives and we hope to deploy our system in museums of Florence in the near future.

\section{References}

1. Baber, C., Bristow, H., Cheng, S.L., Hedley, A., Kuriyama, Y., Lien, M., Pollard, J., Sorrell, P.: Augmenting museums and art galleries. In: Human-Computer Interaction, INTERACT, vol. 1, pp. 439-447 (2001)

2. Bagdanov, A.D., Del Bimbo, A., Landucci, L., Pernici, F.: MNEMOSYNE: Enhancing the museum experience through interactive media and visual profiling. In: Grana, C., Cucchiara, R. (eds.) MM4CH 2011. CCIS, vol. 247, pp. 39-50. Springer, Heidelberg (2012)

3. Bay, H., Fasel, B., Van Gool, L.: Interactive museum guide: Fast and robust recognition of museum objects. In: Proceedings of the First International Workshop on Mobile Vision (May 2006)

4. Bimbo, A.D., Lisanti, G., Masi, I., Pernici, F.: Person detection using temporal and geometric context with a pan tilt zoom camera. In: 2010 20th International Conference on Pattern Recognition (ICPR), pp. 3886-3889. IEEE (2010)

5. Bruns, E., Brombach, B., Zeidler, T., Bimber, O.: Enabling mobile phones to support large-scale museum guidance. IEEE MultiMedia 14(2), 16-25 (2007)

6. Dalal, N., Triggs, B.: Histograms of oriented gradients for human detection. In: IEEE Computer Society Conference on Computer Vision and Pattern Recognition, CVPR 2005, vol. 1, pp. 886-893. IEEE (2005)

7. Hartley, R.I., Zisserman, A.: Multiple View Geometry in Computer Vision, 2nd edn. Cambridge University Press (2004) ISBN: 0521540518

8. Hatala, M., Wakkary, R.: User modeling and semantic technologies in support of a tangible interface. Journal of User Modeling and User Adapted Interaction 15(3-4), 339-380 (2005)

9. Karaman, S., Bagdanov, A.D.: Identity inference: Generalizing person reidentification scenarios. In: Fusiello, A., Murino, V., Cucchiara, R. (eds.) ECCV 2012 Ws/Demos, Part I. LNCS, vol. 7583, pp. 443-452. Springer, Heidelberg (2012)

10. Karaman, S., Lisanti, G., Bagdanov, A.D., Del Bimbo, A.: From re-identification to identity inference: labelling consistency by local similarity constraints. In: Person Re-identification (to appear, 2013)

11. Kuflik, T., Stock, O., Zancanaro, M., Gorfinkel, A., Jbara, S., Kats, S., Sheidin, J., Kashtan, N.: A visitor's guide in an active museum: Presentations, communications, and reflection. Journal on Computing and Cultural Heritage (JOCCH) 3(3), 11 (2011)

12. Wang, Y., Stash, N., Sambeek, R., Schuurmans, Y., Aroyo, L., Schreiber, G., Gorgels, P.: Cultivating personalized museum tours online and on-site. Interdisciplinary Science Reviews 34(2-3), 2-3 (2009) 